\title{
Atención de personas mayores con demencia a través del modelo de atención centrada en la persona en centros residenciales
}

\author{
Marta Sahagún Navarro ${ }^{1}$ \\ Fecha: Recibido octubre 29 de 2014 - Aceptado diciembre 2 de 2014
}

\begin{abstract}
Resumen
En el presente estudio nos centraremos en la atención de las personas mayores con demencia, concretamente en trece, institucionalizadas en residencias a largo plazo. A través de la utilización de la escala de observación sistemática denominada Dementia-Care Mapping (Bradford Dementia Group, 1997; 2005, citado en Brooker \& Surr, 2009) se pretende conocer el contexto en el que se desenvuelven con el fin de facilitar datos concretos al equipo profesional que vela por su atención. El objetivo que se persigue es estudiar cómo mejorar la calidad de vida de las personas mayores con demencia, mediante una atención centrada en la persona, puesto que dada la alteración en la comunicación y su alto nivel de dependencia, entre otros procesos, a menudo impide que se establezcan los apoyos adecuados. Los resultados más relevantes apuntan a una falta de implicación de los participantes con el entorno, lo cual indica una insuficiencia de estímulos que generen estados de bienestar en los usuarios del centro.
\end{abstract}

Palabras clave: personas mayores, demencia, centros residenciales, atención centrada en la persona, Dementia Care Mapping (DCM).

\section{Care of older people with dementia through the model of person-centered care in residential centers}

\begin{abstract}
In this study we will focus on the care of elders with dementia, particularly in thirteen institutionalized long-term residences. Through the use of the scale of systematic observation called Dementia-Care Mapping (Bradford Dementia Group, 1997; 2005, cited in Brooker \& Surr, 2009) we want to know the context in which they operate in order to provide specific information to the professional team that ensures your attention. The objective pursued is to study how to improve the quality of life of elders with dementia through a focus on the person, since given the alterations in communication and high level of dependence, among other processes, often cause an inadequate support. The most relevant results point to a lack of involvement of the participants with the environment, indicating a lack of stimuli that generate welfare states in the center's users.
\end{abstract}

Keywords: elderly, dementia, residential centers, person-centered care, Dementia Care Mapping (DCM).

${ }^{1}$ Trabajadora social, Magíster y Doctoranda en Atención Sociosanitaria a la Dependencia. Docente Investigadora en la Corporación Universitaria del Caribe - CECAR. marta.sahagun@cecar.edu.co 


\section{Introducción}

Actualmente, nos encontramos ante un fenómeno de envejecimiento poblacional a nivel mundial, en el que la esperanza de vida se ha visto incrementada notablemente. España, por ejemplo, se sitúa en una de las tasas con mayor envejecimiento del mundo, sumando un total de 7.632 .925 personas de más de 65 años y 2.123.785 de más de 80, mayoritariamente mujeres (IMSERSO, 2009). Del mismo modo, la esperanza de vida se situaba en 1910 en 42,6 años en la mujer y de 40,9 en los varones, siendo en 2010 de 84,8 en las mujeres y de 78,3 en los varones, situándose así la mujer en la tasa de esperanza de vida más alta de la Unión Europea (IMSERSO, 2009).

Uno de los motivos por el cual se ha logrado alcanzar estas cifras ha sido gracias al desarrollo de la ciencia y la más destacada, las Ciencias Sanitarias, puesto que han permitido que exista un aumento de la longevidad además de un descenso de la mortalidad infantil.

Este aumento de la longevidad ha llevado consigoelaumento desituaciones de dependencia, en el que las personas por razones derivadas de la edad, la enfermedad o la discapacidad, y ligadas a la falta o pérdida de autonomía física, mental, intelectual o sensorial, precisan de apoyos humanos o materiales para realizar las actividades de la vida diaria (IMSERSO, 2009).

No obstante, la protección de las personas en situación de dependencia ha estado influenciada por los cambios en el modelo de familia y en las relaciones de género en las sociedades modernas tras la incorporación de la mujer al mercado laboral, retrasando así la maternidad y reduciendo la descendencia. Tal y como señala Alberdi (1999) "Las demandas de ayuda familiar someten continuamente a las mujeres a tener que optar entre lealtades contrapuestas, obligándoles a elegir entre sus oportunidades individuales y laborales y su solidaridad con las generaciones anteriores".

En este sentido, podemos evidenciar un cambio demográfico que ha planteado un giro en las políticas sociales de las sociedades modernas, acelerando la puesta en marcha de sistemas de cuidados de larga duración. Este hecho ha dado lugar a un largo proceso de demanda social para la creación de una red de centros y recursos sociales para la protección y atención a las personas en situación de dependencia distintas de la familia.

Es aquí donde, en la atención de las personas mayores institucionalizadas, nos encontramos ante un tradicional modelo médico-rehabilitador, en el que la persona mayor es considerada un sujeto pasivo, receptor de atención y apoyo, por por lo que se corre el riesgo de entrar en pautas de deshumanización. Tal y como especifican VilaMiravent, Villar, Celdrán y Fernández (2012) no tener en cuenta los deseos y necesidades de la persona con demencia promueve su pasividad y se compromete incluso su identidad como persona.

Asimismo, promover la pasividad de la persona mayor desencadena un fenómeno definido por Baltes y Baltes (1990 citado en Montorio, 2007) como exceso de incapacidad, el cual hace alusión "al deterioro provocado por el desuso de las capacidades preservadas, que no son consecuencia directa de un estado de fragilidad física o enfermedad y que son de carácter reversible" (p.50).

Es, en este punto, donde entra en juego el modelo de atención centrada en la persona (en adelante, ACP) desarrollado por Kitwood y Bredin (1992, citado en Brooker \& Surr, 2009), en el que la persona mayor con demencia institucionalizada pasa a ser un agente activo, y plantea que una de las máximas prioridades es mejorar su atención, empleando una valoración individualizada, para que se mejore su calidad de vida.

En este sentido, Kitwood y Bredin (1992, citados en Brooker \& Surr, 2009) señalan que, para comprender los comportamientos de una persona con demencia y poder así realizar una buena atención, existen factores que influyen en la manera de actuar, sentir y pensar de las mismas, además del daño neurológico producido. 
BÚSQUEDA - Julio / Diciembre de 2014 - No. 13 (82 - 99)

De esta forma, encontramos aspectos relacionados con su salud y el estado físico en general, en el cual debemos ser capaces de intervenir sin menoscabar las capacidades que aún se conservan.

También, es de suma importancia conocer las experiencias pasadas de la persona con demencia, su biografía e historia de vida, puesto que pueden ayudar a los miembros del personal a comprender conductas confusas. Por ejemplo, si una persona ha ocupado puestos de dirección durante toda su vida laboral es muy probable que quiera sentarse en la oficina para revisar documentos..., y seguramente le resultará desconcertante que un cuidador, al que ve como un subordinado, le diga lo que tiene que hacer.

Asimismo, un factor determinante es la personalidad y los rasgos característicos de la misma, puesto que influyen en la manera de enfrentarse a la demencia. Por ejemplo, una persona que le gusta controlarlo, todo seguramente tendrá más dificultades en aceptar las consecuencias de la demencia, que alguien que siempre ha delegado sus decisiones en los demás.

Por último, debemos tener en cuenta que a medida que se van perdiendo las capacidades verbales, el contacto humano, cariñoso y cálido se vuelve más importante a través de los canales no verbales, lo cual resalta la importancia de las relaciones interpersonales de la persona con demencia, teniendo presentes las características de su entorno. Todo este conjunto de aspectos Tom Kitwood los agrupó creando el modelo enriquecido de la demencia (citado en Brooker \& Surr, 2009).

Asimismo, el modelo de atención centrada en la persona identifica cinco necesidades psicológicas que toda persona posee en mayor o menor medida pero que, en el caso de estar presente la demencia, es responsabilidad de los profesionales y cuidadores profesionales ofrecer su cobertura. Son las siguientes (Brooker \& Surr, 2009): a) Confort, referido al suministro de cariño, atención y proximidad hacia los demás, evitando intimidaciones y omisión de cuidados; b) identidad, que tiene que ver con el respeto hacia la persona, independientemente de su experiencia y edad, aceptación del participante y refuerzo a las habilidades que aún se conservan, evitando infantilizar a la persona mayor, etiquetarla o infravalorarla; c) apego, definido como el reconocimiento de los participantes como únicos y la sensibilidad por los sentimientos y emociones, para fomentar vínculos de afecto y confianza, y evitar la culpa a los participantes por cosas que no han podido hacer, manipulaciones para que hagan o dejen de hacer alguna cosa, ningunearles; d) ocupación, en el sentido de empoderar las capacidades de los participantes, facilitándoles el apoyo necesario, colaborando conjuntamente para cubrir sus necesidades, evitando imponer, cosificar, interrumpir en la realización de actividades; e) inclusión, tanto física como psicológica, formando parte de un grupo, pudiendo acceder al ocio, sin estigmatizar, ignorar ni humillar, teniendo en cuenta que las personas con demencia corren un gran riesgo de estar socialmente aisladas aunque residan en un entorno comunitario.

En la medida en la que estas necesidades psicológicas no son atendidas, se puede producir un proceso de deshumanización, en el que los deseos y necesidades de las personas con demencia se encuentren mermados.

De este modo, Kitwood (1992, citado en Brooker \& Surr, 2009) denominó psicología social maligna (en adelante, PSM) a estos riesgos de la práctica profesional que producen un entorno y una cultura puramente asistencialista y paternalista, en las que se despersonaliza a la persona con demencia, reforzando entornos poco participativos, es cuando el malestar de la persona que no está siendo atendida de forma integral incrementa, disminuyendo así su bienestar.

Asimismo, la PSM recoge según Kitwood(1992, citado en Brooker \& Surr, 2009) la monitorización de niveles de bienestar o malestar emocional experimentado junto a veintitrés categorías de diferentes tipos de comportamientos que puede haber tenido el participante en intervalos de tiempo de cinco minutos. Esta evaluación se realiza a 
través de un seguimiento continuado entre cinco y ocho personas con demencia (participantes) durante un periodo de tiempo representativo (seis horas durante el periodo de mañana-tarde es lo recomendable, pero no hay datos empíricos que verifiquen la representatividad de este periodo de tiempo²).

Todos estos elementos anteriormente citados han conformado la denominada escala de observación sistemática Dementia-Care Mapping (Bradford Dementia Group, 1997; 2005), una herramienta diseñada por los psicólogos Tom Kitwood y Kathleen Bredin de la Universidad de Bradford (Inglaterra) con el fin de determinar la calidad de vida $^{3}$ y de atención que recibían las personas con demencia en entornos asistenciales especializados, así como sus familias, a través de formación, estudios, investigación y el reconocimiento del papel primordial que desempeñan los profesionales.

En este sentido, una de la propuestas que se viene planteando hace años es la de priorizar a la persona frente a la demencia (Fernández \& Vila, 2006), de tal forma que para obtener información referente a la situación de una persona acerca del grado de autonomía con la que ésta hace frente a su vida cotidiana, es necesario valorar la mayor parte de áreas en las que se desenvuelve el individuo. Es decir, no se trata sólo de medir el estado de salud o la memoria, sino de valorar su posibilidad de autonomía en cada área y además, relacionarlo con otras: física, provisión de servicios, apoyo social, contexto, etc., a fin de tener una idea global e interrelacionada del funcionamiento y la autonomía de esa persona en su vida cotidiana (Julve, 2011).

Sin embargo, tal y como especifica Fernández y Vila (2006) la dificultad emerge en cómo obtener la opinión, la percepción subjetiva y, en definitiva, la valoración de los propios interesados. Éstos, a causa del deterioro cognitivo de la enfermedad, no la pueden expresar.
Por ello, esta investigación trata de dar a conocer posibles medidas para una adecuada intervención a través de la observación sistemática de personas mayores con demencia institucionalizadas que, dada su alteración en la comunicación y su alto nivel de dependencia, entre otros procesos, a menudo impide que se establezcan los apoyos adecuados.

De este modo, la metodología que nos propone este instrumento es la de observar detenidamente las conductas de la persona con demencia institucionalizada a través de una serie de indicadores que, en primera instancia, son observados por distintos profesionales (también llamados mapeadores) para precisar e indicar hasta qué punto dos mapeadores codifican igual al observar a los mismos participantes. Tal como recomienda el manual de DCM (Bradford Dementia Group, 2005, citado en Brooker y Surr, 2009) si no se alcanza un nivel de acuerdo, de al menos un $80 \%$, se debe volver a realizar una nueva observación hasta llegar a dicha cifra de fiabilidad. Todo ello con el fin de agilizar el proceso de observación.

Una vez realizada esta prueba de fiabilidad, se procede a la toma de datos reales, siendo observaciones exhaustivas y en tiempo real, a lo largo de varias horas, teniendo en cuenta el modelo teórico de la atención centrada en la persona. Este modelo implica valorar a las personas con independencia de su edad y de su estado de salud, además de individualizar y priorizar la perspectiva de la persona con demencia, reconociendo la importancia de las relaciones sociales (Brooker \& Surr, 2009).

Tras la recogida de datos y análisis de los resultados obtenidos, uno de los objetivos que se plantean es la promoción de la autonomía de las personas mayores con demencia para ayudar a fomentar la realización de actividades, a través de los datos facilitados; para poder prevenir así situaciones de dependencia, valorar su

Un estudio de Estados Unidos no publicado (Douglass y Johnson, 2002, citado en Brooker, 2006) demostró que había niveles aceptables de fiabilidad inter-observador en mapeos de más de 4 horas de duración.

${ }^{3}$ Entendiendo calidad de vida como el sentimiento de satisfacción de la persona con demencia en áreas como el bienestar físico, el emocional y las relaciones personales. 
autodeterminación y con esto, mejorar su calidad de vida. Todo ello, con el fin de ofrecer apoyo al centro residencial para establecer un plan de atención individualizado junto a un periodo de seguimiento.

Asimismo, se pretende reflejar la funcionalidad que tiene este método para hacer posible su uso en las organizaciones de carácter asistencial (residencias, hospitales y centros de día) con el fin de incidir positivamente en la calidad de vida y atención de las personas con demencia.

\section{Metodología}

\section{Participantes}

Los participantes fueron 13 personas en situación de dependencia psicofuncional, todas ellas mujeres mayores de 65 años diagnosticadas con demencia, alguna de ellas con Alzheimer en fase avanzada. Todos los participantes pertenecen a una misma institución, que es un centro público con gestión privada, y con un tiempo superior de ingreso de más de 6 meses.

Previamente a la observación, se solicitó la firma de consentimientos de los participantes (o de sus familiares) y la de los profesionales involucrados.

Estas usuarias fueron objeto de este estudio por la necesidad que presentaban de una atención individual y personalizada, puesto que generalmente se desconoce cómo actuar ante situaciones de demencia, sobre todo en deterioros cognitivos graves tales como los que se presentan en este estudio, siendo la farmacología el tratamiento estrella para la misma. Ello hace necesaria focalizar los esfuerzos en intentar llevar a cabo también una terapia no farmacológica, pues está demostrado que en personas con demencia leve-moderada se puede llevar a cabo ambas terapias (farmacológica y no farmacológica).
Los objetivos a largo plazo que se pretenden conseguir con esta toma de datos, desde el centro residencial como método futuro de prevención, es abordar un plan de atención integral desde lo cognitivo, conductual y funcional que ayude a mantener el estado cognitivo de la persona con demencia e interrumpa los procesos involutivos característicos de la misma.

\section{Instrumento}

El instrumento utilizado para la recolección de datos fue la escala Dementia Care-Mapping, en su versión octava (la más actual). Según el protocolo de evaluación del DCM (Bradford Dementia Group, 2009), para analizar los resultados se debe elaborar un informe individual para cada participante observado, el cual debe contener:

1. Datos sobre ánimo/implicación;

2. Datossobrelacategoría del comportamiento;

3. Detractores personales;

4. Potenciadores personales;

5. Otras observaciones importantes para la evaluación.

El primer tipo de información registrada son los datos sobre ánimo/implicación (Mood and Engagement; $M E$ ) que describen el estado de bienestar o malestar (Well/ill-being scores; $W I B)$ que los participantes han experimentado en cada intervalo de tiempo (IT en adelante), representándose en una escala de seis puntos $(+5,+3,+1,-1,-3,-5)$, siendo el -5 el máximo nivel de malestar y el +5 el máximo nivel de bienestar, siguiendo los criterios que se muestran en la tabla 1.

La combinación e interpretación de los datos ME de cada IT permite valorar el nivel de bienestar/ malestar (WIB) de la persona con demencia. Sin embargo, para realizar este cálculo se deben tener datos sobre los valores ME a lo largo de, por lo menos, cuatro horas o $48 \mathrm{IT}$.

"Brooker y Surr (2009) hablan de bienestar "relativo" dado que "existe un consenso de que no se puede considerar que las personas con demencia experimenten el bienestar exactamente igual que las personas que no la tienen, ya que la demencia conlleva la pérdida de capacidades cognitivas, además de suavizar o acentuar la expresión de las emociones". Por ello, concluyen que una persona con demencia experimentará bienestar relativo cuando se presenten más indicadores de bienestar que de malestar en un espacio de tiempo determinado, centrando los cuidados y la atención en las necesidades, habilidades y preferencias de la persona. 
Marta Sahagún Navarro - Atención de personas mayores con demencia a persona en centros residenciales

Tabla 1. Niveles de bienestar y malestar emocional (ME) según el DCM

\begin{tabular}{lcl}
\multicolumn{1}{c}{ Ánimo } & Valor ME & \multicolumn{1}{c}{ Implicación } \\
\hline $\begin{array}{l}\text { Muy contentos, alegres. Estado de ánimo } \\
\text { muy positivo. }\end{array}$ & +5 & $\begin{array}{l}\text { Profundamente ensimismados. Muy } \\
\text { entretenidos. }\end{array}$ \\
\hline $\begin{array}{l}\text { Contentos, alegres, relajados. Estado de } \\
\text { ánimo considerablemente positivo. }\end{array}$ & +3 & $\begin{array}{l}\text { Concentrado pero fácil de distraer. } \\
\text { Muy ensimismados. }\end{array}$ \\
\hline $\begin{array}{l}\text { Neutral. Ausencia de señales evidentes } \\
\text { de ánimo positivo o negativo. }\end{array}$ & +1 & $\begin{array}{l}\text { Atentos y conscientes del entorno. } \\
\text { Participación breve o intermitente. }\end{array}$ \\
\hline $\begin{array}{l}\text { Pequeñas señales de estado de ánimo } \\
\text { negativo. }\end{array}$ & -1 & Ausente y sin contacto. \\
\hline $\begin{array}{l}\text { Señales considerables de estado de } \\
\text { ánimo negativo. }\end{array}$ & -3 & \\
\hline $\begin{array}{l}\text { Muy afligido. Señales claras de estado de } \\
\text { ánimo muy negativo. }\end{array}$ & -5 & \\
\hline
\end{tabular}

Fuente: Brooker y Surr, 2009

Asimismo, la ACP pone mucho énfasis en la conservación de la persona en el contexto de las relaciones personales para mantener un bienestar relativo ${ }^{4}$ en la demencia. Por lo tanto, uno de los indicadores de calidad de la atención en una organización puede ser la cantidad de tiempo que los participantes pasan en un estado de ánimo o implicación negativos de forma ininterrumpida (UNME en adelante). Esto se detecta estudiando las hojas de datos en bruto durante periodos de seis IT consecutivos pasados con valores ME negativos.

El segundo tipo son los datos sobre la categoría del comportamiento (Behaviour Category Code; $B C C$ ) donde se recogen veintitrés diferentes tipos de comportamiento que son más frecuentes en un entorno residencial y que pudo haber tenido el participante. Para memorizarlos se identifican con una palabra que ayuda a recordar la letra del código, así como una breve descripción de la categoría, tal y como podemos observar en la tabla 2. No se incluyen los IT en los que el mapeador interactuó con el participante, pero sí aquellos en los que se ha registrado un BCC sin un valor ME. Pueden presentarse los resultados en formato gráfico.
Cuando el mapeador abandona el lugar de observación y no puede proceder con la misma, puede codificarse con la letra $Q$.

También, este conjunto de códigos permite identificar una serie de indicadores de la calidad de vida de la persona institucionalizada. Son los siguientes:

Por un lado, el potencial de implicación positiva (IPO) existente en el participante durante la observación, teniéndose en cuenta las siguientes categorías de gran potencial: $A, D, E$, F, G, I, J, K, L, O, P, R, S, T, V e Y.

Por otro lado, se recoge la diversidad ocupacional (DO) que ha tenido el participante durante la observación, teniendo en cuenta las preferencias y capacidades de implicación de los participantes en las siguientes categorías de gran potencial: A, D, E, G, I, J, K, L, O, R, S, T, V e $\mathrm{Y}$, con un valor ME positivo de un $2 \%$ o más, lo cual es la suma de los distintos comportamientos que ha tenido con un valor máximo de 14 , puesto que este es el número máximo considerado para esta variable. 
BÚSQUEDA - Julio / Diciembre de 2014 - No. 13 (82 - 99)

Tabla 2. Códigos de categorías del comportamiento

\begin{tabular}{|c|c|c|}
\hline Código & Palabra clave & Descripción general \\
\hline A & Interacción & $\begin{array}{l}\text { Interacción con otros, verbalmente o no, sin otra actividad } \\
\text { observable. }\end{array}$ \\
\hline B & Borderline / Límite & Implicación social, pero pasiva (contemplación). \\
\hline $\mathrm{C}$ & Desvinculado & Socialmente desvinculado, aislado. \\
\hline $\mathrm{D}$ & Dedicación / Autocuidado & Atención a uno mismo. \\
\hline E & Expresiva & Actividades expresivas o creativas. \\
\hline $\mathrm{F}$ & Ingesta & Comer, beber. \\
\hline G & Recuerdo & Reminiscencia y revisión de la propia vida. \\
\hline 1 & Intelectual & Actividades que priorizan las capacidades intelectuales. \\
\hline J & Actividad física & Hacer ejercicio o deporte. \\
\hline K & Caminar & Caminar, estar de pie, moverse con independencia. \\
\hline $\mathrm{L}$ & Ocio & Ocio, diversión, actividades recreativas. \\
\hline N & Dormir & Dormir, descansar. \\
\hline $\mathrm{O}$ & Objetos & Mostrar afecto o relación con objetos inanimados. \\
\hline$P$ & Asistencia física / Práctica & Recibir asistencia práctica, física o personal. \\
\hline $\mathrm{R}$ & Religiosidad & Participar en una actividad religiosa. \\
\hline S & Sexualidad & Expresión sexual. \\
\hline T & Sensaciones sensoriales & Estimulación directa de los sentidos. \\
\hline$U$ & Sin respuesta & Intentar comunicarse y no recibir respuesta (chillar, llorar). \\
\hline V & Vocacional & Participar en una actividad, taller. \\
\hline W & Autoestimulación repetitiva & Autoestimulación repetitiva (balancearse). \\
\hline$x$ & Excreción & Episodios asociados con la excreción. \\
\hline Y & Yo mismo & $\begin{array}{l}\text { Interacción en ausencia de otra persona observable } \\
\text { (hablar solo). }\end{array}$ \\
\hline Z & Cero & $\begin{array}{l}\text { Cuando no encaja en ninguna de las categorías } \\
\text { precedentes. }\end{array}$ \\
\hline
\end{tabular}


Marta Sahagún Navarro - Atención de personas mayores con demencia a persona en centros residenciales

Del mismo modo, encontramos las variables de agitación y angustia (AGI) a partir de la cantidad de IT pasados en la categoría $U, K, W$, e $Y$ en valores $M E$ negativos $(-1,-3,-5)$, mostrando el malestar emocional del participante.

Otro aspecto que contempla es el abandono o aislamiento (ABA) que se constituye por la cantidad de tiempo pasado en las categorías $\mathrm{C}$ y $\mathrm{N}$, en las que el participante no muestra señales de implicación consigo mismo ni con el mundo que lo rodea, tanto activa como pasivamente. Generalmente, las personas con demencia tienen reducidas las capacidades de implicarse de forma autónoma con los demás. Por ello, el entorno en el que se desenvuelve el participante o los objetos que se encuentran en él pueden ser la consecuencia de un bienestar reducido si no se ofrecen los apoyos necesarios para la implicación.

Por último, se tiene en cuenta la implicación pasiva (IPA), identificándose con la categoría B. Sería importante comentar con el personal, maneras de convertir esa implicación pasiva en activa y garantizar que no se convierta en una falta de implicación.

El tercer tipo de información registrada como otro indicador de la calidad de la atención proporcionada en la organización es la presencia de detractores personales (Personal Detractions; PD) o potenciadores personales (Personal Enhancers; PE), una manera de captar la calidad de las interacciones entre el personal y los participantes observados.

Los PD son comportamientos de los miembros del personal que podrían resultar perjudiciales para la persona con demencia y mina una o más de sus necesidades psicosociales antecitadas (confort, apego, identidad, ocupación e inclusión). Por ejemplo, hablar del residente en su presencia como si este no estuviera, se catalogaría como "ignorar", y minaría la necesidad psicosocial de inclusión de una persona.

Sin embargo, los PE son conductas de los miembros del personal consideradas como positivas para preservar una o más de las necesidades psicosociales de la persona. Por ejemplo, dirigir unas palabras de apoyo para animar a una persona con demencia a hacer una actividad por ella misma, se codificaría como "capacitar" y cubriría la necesidad de ocupación de la persona.

Por último, se tienen en cuenta otras observaciones importantes que el mapeador registra en la hoja de observación, puesto que ayudan a interpretar los datos y a redactar un informe final de los resultados. Es importante incluir información acerca de lo que están haciendo los participantes durante cada IT y las sensaciones sobre el entorno en su conjunto.

De esta forma, la observación sistemática se realizaba cada cinco minutos, en ella el mapeador registraba el comportamiento (BCC) y el nivel de malestar-bienestar (ME) que se daba en ese momento. A lavez, se registraban los potenciadores y detractores personales que se observaban.

El periodo de observación se realizó en un intervalo de entre mínimo 2 horas y un máximo de 4 horas consecutivas, durante el horario de 9:00 horas de la mañana a 16:00 horas de la tarde, en días laborables de lunes a viernes. La recogida de datos se realizó durante el periodo de abril y mayo de 2011.

\section{Resultados}

Una vez expuestos los apartados que componen un análisis de datos de DCM se exponen a continuación los informes individuales realizados a cada participante.

\section{a) Resumen individual participante 1:}

Comentarios sobre los estados de ánimo e implicación, los comportamientos y el bienestar: Esta participante pasó gran parte del día (45\%) hablando sola (categoría Y). Comentaba que "tenía que ir a por las vacas". Además, estuvo el $23 \%$ del día implicada pasivamente (B), otro $23 \%$ comiendo y un $9 \%$ recibiendo asistencia práctica. Por ejemplo, ella misma no podía cortar la carne que tenía para comer (no sabe usar 
tenedor y cuchillo) y una auxiliar de enfermería (AE en adelante) lo hizo. Asimismo, pasó el 82\% del día en un estado de ánimo e implicación positiva, siendo el $18 \%$ en un nivel +3 .

Observaciones generales para el personal: La participante pasó parte de la mañana con un potencial de implicación positiva del $77 \%$, en las categorías de potencial alto de ingesta $(F)$, asistencia física-práctica $(P)$ e interacción sin la presencia de otra persona $(Y)$. En este periodo observacional estuvo muy poco tiempo implicada en otra actividad que no fuera comer, recibir asistencia o hablar sola. La diversidad ocupacional fue tan solo de 1, en la categoría Y, por lo que ¿Podemos pensar en otras actividades que la impliquen más durante el día? Los mayores niveles de valores ME positivos han transcurrido cuando la participante estaba comiendo. Para las próximas observaciones sería interesante valorar el momento de la comida así como el contenido de ésta, con tal de apreciar si el estado positivo de +3 es porque va a comer o porque el plato de ese día le es especialmente agradable. Además, valoraría el grado de empatía que se observa entre la usuaria y las auxiliares.

\section{b) Resumen individual participante 2:}

Comentarios sobre los estados de ánimo e implicación, los comportamientos y el bienestar: La participante pasó gran parte del día (52\%) durmiendo $(N)$. Después de que llevara diez minutos durmiendo, se despertó y la $A E$ la peinó y le puso colonia. Asimismo, estuvo el 14\% implicada pasivamente (B) y otro $14 \%$ recibiendo asistencia práctica $(P)$, en el que le facilitan la medicación. Además, pasó un 10\% comiendo $(F)$, un $5 \%$ deambulando $(K)$ para llegar a su sala y otro $5 \%$ participando en una tarea $(V)$, la de limpiar la mesa tras la comida. También, estuvo el $60 \%$ del día en un estado de ánimo e implicación positiva y el $30 \%$ en un nivel +3 . Sin embargo, pasó en un estado de ánimo e implicación negativo el $10 \%$ del día.

Observaciones generales para el personal: La participante tuvo durante la mañana un potencial de implicación positiva del 29\% en categorías de potencial alto, tales como comer $(F)$, recibir asistencia personal $(P)$ y participación en la tarea (V) mencionada anteriormente. La diversidad ocupacional en este periodo de observación fue de 2, en el que se incluyen las categorías $\mathrm{K}$ y $\mathrm{V}$. Sin embargo, la participante tuvo un aislamiento del $52 \%$ pasado en la categoría N (dormir). Este periodo se vio interrumpido brevemente por una asistencia personal que no dificultó que siguiera durmiendo. Como hemos mencionado anteriormente, las personas con demencia tienen reducidas las capacidades de implicarse de forma autónoma con los demás, por lo que es muy importante que su entorno le ofrezca los apoyos adecuados para su implicación. Del mismo modo que la primera participante, ¿podríamos pensar en otras actividades que la impliquen más durante el día? Así, reduciríamos el número de experiencias pasadas en "N" durante esta parte del día. Volviendo a la asistencia personal que la AE ofreció a la participante (peinarla y ponerle colonia, $\mathrm{P}-1$ ) es una acción que infantiliza a la persona y daña su autodeterminación. Los mayores niveles de valores ME positivos han transcurrido cuando la participante comía y limpiaba la mesa. Tras los resultados obtenidos, sería favorable fomentar activamente la realización de actividades para que, de este modo, pudiera encontrarse en un índice de bienestar considerablemente positivo. Por otro lado, para las próximas observaciones sería interesante valorar si la participante aprecia el momento de la comida o es la comida en sí. De este modo, podríamos conocer cuál es la dieta que más le gusta.

\section{c) Resumen individual participante 3:}

Comentarios sobre los estados de ánimo e implicación, los comportamientos y el bienestar: La participante pasó la mayoría de tiempo (77\%) durmiendo ( $N$ ) y el 23\% comiendo y bebiendo ( $F$ ) a desgana. Además, estuvo el $80 \%$ del día en un estado de ánimo e implicación negativo y el 20\% en un nivel -3 .

Observaciones generales para el personal: La participante tuvo un aislamiento del $77 \%$ pasado en la categoría $N$ (dormir). No obstante, este periodo fue interrumpido dos veces para beber y comer $(F)$, un comportamiento de un $23 \%$ de potencial de implicación positiva. Sin embargo, el 
Marta Sahagún Navarro - Atención de personas mayores con demencia a persona en centros residenciales

periodo de ingesta lo pasó en un estado de ánimo e implicación negativo. ¿Sabemos qué provocó esta situación? ¿Podría ser debido a una falta de descanso que le impide encontrarse en bienestar? O bien ¿Por una falta de implicación con el entorno que no ayuda a mantenerse despierto? ¿O quizás porque no tenía sed ni hambre? O incluso ¿Podría encontrarse en un estado de depresión? Por otro lado, sería favorable reducir la cantidad de códigos "N" (dormir).

\section{d) Resumen individual participante 4:}

Comentarios sobre los estados de ánimo e implicación, los comportamientos y el bienestar: La participante pasó el $41 \%$ implicada pasivamente (B). La AE le colocó una almohada en la butaca para un mayor confort. No obstante, estuvo el $18 \%$ del día desvinculada (C). Por otro lado, estuvo otro $18 \%$ del día comiendo $(F)$, un $14 \%$ con estimulación directa de los sentidos (T) y el $9 \%$ durmiendo (N). Pasó el $80 \%$ del día en un estado de ánimo e implicación positivo. Sin embargo, el $20 \%$ del día se encontró en un estado de ánimo e implicación negativo.

Observaciones generales para el personal: La participante pasó un potencial de implicación positiva del $32 \%$ en las categorías de ingesta (F) y estimulación directa de los sentidos ( $T$ ). Su diversidad ocupacional tan sólo fue de 1 , pasado en la categoría T. No obstante, tuvo un aislamiento del $27 \%$ pasado en la categoría C (18\%) y $N(9 \%)$. Los mayores niveles de valores $\mathrm{ME}$ positivos transcurrieron cuando estaba implicada pasivamente, cuando comía y cuando se estimulaban sus sentidos. En cambio, los mayores niveles de valores ME negativos ocurrieron cuando se encontraba totalmente desvinculada del entorno. La participante estuvo alternando las categorías B y C, lo que nos suscita a pensar que estar implicada pasivamente durante un periodo de tiempo hace que se acabe desvinculando. Por ello, ¿Se podría trabajar más en fomentar categorías como la T para su mayor implicación con el entorno? Si en las próximas observaciones en las que la participante está siendo estimulada durante más periodos de tiempo se sigue desvinculando, podríamos pensar en una posible depresión.

\section{e) Resumen individual participante 5:}

Comentarios sobre los estados de ánimo e implicación, los comportamientos y el bienestar: La participante pasó gran parte del día (36\%) implicada pasivamente (B). Sería favorable reducir la cantidad de códigos "B" pasados en esta categoría. Además, la participante pasó el $18 \%$ implicada con un objeto inanimado $(\mathrm{O})$, en concreto con una servilleta y un muñeco. De este modo, estuvo el $82 \%$ del día en un estado de ánimo e implicación positiva y el 5\% en un nivel +3 . Sin embargo, tuvo un estado de ánimo e implicación negativo durante el 14\% del día.

Observaciones generales para el personal: La participante estuvo en un potencial de implicación positiva del $46 \%$ en las categorías A (interacción con los demás), $F$ (ingesta), O (manipulación de objetos) y T (estimulaciones sensoriales). Su diversidad ocupacional fue de 3 , en las categorías A, O y T. No obstante, tuvo un aislamiento del 9\% pasado en la categoría C (desvinculada). Quizás cambiándole el objeto o estimulándole los sentidos pudiéramos evitar estos estados. Los mayores niveles de valores ME positivos han transcurrido cuando la participante manipulaba un muñeco, por lo que ¿podemos facilitarle más objetos que pueda manipular? Por otro lado, los mayores niveles de valores ME negativos transcurrieron cuando la participante estaba desvinculada (C) y cuando cogió la almohada de su compañera y esta le pegó (A-1). No obstante, habría que valorar el acto que tuvo la participante de coger una almohada voluntariamente, lo que indica que existe una capacidad de autodeterminación que habría que promover.

\section{f) Resumen individual participante 6:}

Comentarios sobre los estados de ánimo e implicación, los comportamientos y el bienestar: La participante 6 pasó el $24 \%$ del día desvinculada (C), otro $24 \%$ durmiendo (N) y un $20 \%$ implicada pasivamente (B). Por otro lado, estuvo el $12 \%$ del día comiendo $(F)$, el $8 \%$ recibiendo asistencia sanitaria $(P)$, en concreto una, donde la $A E$ le limpió los ojos; un 4\% interaccionando con otros (A), específicamente la AE la despertó diciendo que "durante el día no se duerme"; otro 4\% comunicándose sin recibir respuesta y otro $4 \%$ 
con episodios relacionados con la excreción. Así, la participante estuvo el 53\% del día en un estado de ánimo e implicación positiva y el $5 \%$ en un nivel +3 . Sin embargo, estuvo el $42 \%$ del día en un estado de ánimo e implicación negativo.

Observaciones generales para el personal: La participante estuvo un $24 \%$ en un potencial de implicación positiva en las categorías A (interacción con los demás), F (ingesta) y P (asistencia sanitaria). No obstante, estuvo en un estado de agitación y angustia del $4 \%$ en la categoría U (intentándose comunicar con los demás sin recibir respuesta). Además, tuvo un aislamiento del $48 \%$ pasados en las categorías C (24\%) y N (24\%). Los mayores niveles de valores ME positivos han transcurrido cuando se acercó un trabajador y ella le sonrió. Además, en dos ocasiones habló con el mapeador, lo que puede indicar que a la participante le gusta relacionarse con los demás y busca la forma de hacerlo. ¿Se podría fomentar más este tipo de relaciones personales para un mayor bienestar, reduciendo así experiencias en las categorías $\mathrm{C}, \mathrm{N}$ y $\mathrm{B}$ ? Los mayores niveles de valores $\mathrm{ME}$ negativos han ocurrido cuando la participante estaba desvinculada (C), cuando la $A E$ la despertó para comer (A-1) y cuando intentaba comunicarse sin recibir respuesta $(U)$. Por un lado, una vez más podemos observar que la falta de implicación de los usuarios conlleva a su desvinculación con el entorno y posteriormente, a quedarse dormidos. Por otro lado, observamos que en el intento de comunicarse con las auxiliares posteriormente, recibe asistencia y después, pasa por un periodo relacionado con la excreción. ¿Es adecuado no dar una respuesta cuando se ha intentado comunicar una usuaria con una AE?

\section{g) Resumen individual participante 7:}

Comentarios sobre los estados de ánimo e implicación, los comportamientos y el bienestar: La participante pasó gran parte del día (69\%) durmiendo (N). Además, pasó el 14\% desvinculada (C), un 10\% implicada pasivamente (B), dado que seguía con la mirada a la AE y un $7 \%$ comiendo ( $F$ ). La participante estuvo el 56\% del día en un estado de ánimo e implicación positivo. No obstante, tuvo un estado de ánimo e implicación negativo el $44 \%$ del día.
Observaciones generales para el personal: La participante tuvo un potencial de implicación positiva del $7 \%$ en la categoría $F$ (ingesta). Sin embargo, tuvo un $83 \%$ de aislamiento en las categorías C (14\%) y N (69\%). ¿A qué se debe tantas horas de sueño? ¿No descansa? ¿Falta de estimulación? O incluso ¿un déficit en el estado anímico? Los mayores niveles de valores ME positivos han transcurrido implicada pasivamente y comiendo. Sin embargo, los mayores niveles de valores ME negativos han ocurrido cuando la participante estaba desvinculada. ¿Se podría intervenir para trabajar su implicación con el entorno? Si necesita ser estimulada ¿por qué no se hace? Sus capacidades están completamente en desuso, por lo que está desvinculada durante el día.

\section{h) Resumen individual participante 8:}

Comentarios sobre los estados de ánimo e implicación, los comportamientos y el bienestar: La participante pasó gran parte del día (76\%) durmiendo (N). También, estuvo el 10\% del día comiendo, otro $10 \%$ con una autoestimulación repetitiva (W); concretamente se frotaba las manos, y un 3\% desvinculada (C). Asimismo, estuvo el $71 \%$ del día en un estado de ánimo e implicación positivo. Sin embargo, tuvo un estado de ánimo e implicación negativo el 29\% del día.

Observaciones generales para el personal: La participante solamente tuvo un potencial de implicación positiva del 10\% en F (ingesta), por lo que no tiene ningún tipo de diversidad ocupacional. Por otro lado, tuvo un estado de agitación y angustia del 3\% en W (autoestimulación repetitiva). ¿Se podría intervenir para evitar este estado de ansiedad? ¿Frotarse las manos es un acto comunicativo no verbal que indica cierto grado de nerviosismo? Asimismo, estuvo en un aislamiento del $76 \%$ pasado en la categoría N (dormir), por lo que ¿Podemos intervenir estimulándola para que esté más implicada con el entorno? ¿Debemos valorar tanto tiempo de sueño como un posible estado de depresión o simple aburrimiento? Los mayores niveles de valores ME positivos han transcurrido en un IT de autoestimulación repetitiva positiva y comiendo. Los mayores niveles de valores ME negativos han ocurrido cuando se 
Marta Sahagún Navarro - Atención de personas mayores con demencia a persona en centros residenciales

ha encontrado desvinculada y en un estado de ansiedad, frotándose las manos. Podemos observar cómo sus capacidades están en desuso, por lo que deberíamos intervenir estimulándola.

\section{i) Resumen individual participante 9:}

Comentarios sobre los estados de ánimo e implicación, los comportamientos y el bienestar: La participante pasó gran parte del día (32\%) desvinculada (C) y un $23 \%$ implicada con un objeto inanimado $(\mathrm{O})$. Asimismo, estuvo el $14 \%$ del día comiendo $(F)$, un $9 \%$ interaccionando con otros $(A)$, aunque en una ocasión intentó morder a la auxiliar; otro 9\% durmiendo; otro $9 \%$ con autoestimulación repetitiva, en el que se tocaba el pelo constantemente, y un $5 \%$ con episodios relacionados con la excreción. La participante tan solo pasó el 5\% del día en un estado de ánimo e implicación positivo, siendo el 80\% un estado de ánimo e implicación negativo, del cual un $15 \%$ fue en un nivel -3 . Esta participante registró 2 periodos ininterrumpidos de valores $\mathrm{ME}$ negativos (UNME), de los cuales el tercero fue interrumpido por quedarse dormida.

Observaciones generales para el personal: La participante estuvo en un potencial de implicación positiva del $46 \%$ en las categorías A (interacción con los demás), $F$ (ingesta) y $O$ (manipulación de objetos). En relación con la comida, debemos destacar que si ella misma sabe comer sola, no quiere pechero y no le gusta que le den la comida, hay que respetar su decisión y poner las herramientas necesarias para que ella misma pueda hacerlo sin la necesidad de intervenir. Asimismo, si no le gusta el triturado, ¿podríamos pensar en otro tipo de alimento? Como por ejemplo, una sopa vichisuá. Por otro lado, tuvo un estado de agitación y angustia del 9\% en W (autoestimulación repetitiva). En la observación estaba muy inquieta sentada en el sofá, por lo que ¿La falta de movilidad podría ser la causa de su malestar? ¿Demasiado tiempo en el sofá? ¿Aburrimiento? La participante estuvo casi tres veces en un periodo prologando de valores ME negativos e incluso intentó morder a la auxiliar. ¿Sabemos qué ha provocado esta situación? ¿Se podría intervenir antes para evitar este tipo de espirales negativas de agitación y angustia?
Asimismo, tuvo un aislamiento del $41 \%$ pasado en las categorías C (32\%) y N (9\%). Dado que no puede mantener la atención en la realización de actividades y estar sentada en el sofá le genera mucha inquietud ¿Podemos pensar en otro tipo de ocupación que la implique más de forma activa? Por ejemplo, deambular por el jardín.

\section{j) Resumen individual participante 10:}

Comentarios sobre los estados de ánimo e implicación, los comportamientos y el bienestar: La participante pasó gran parte del día (73\%) hablando sola $(\mathrm{Y})$. Tiene alucinaciones visuales y auditivas constantes. También, pasó el 18\% comiendo $(F)$, un 5\% recibiendo asistencia $(P)$ y otro $5 \%$ con estimulación directa de los sentidos $(T)$, concretamente la $A E$ se le acercó y le dio un beso. Además, estuvo interactuando con su compañera, pero sin recibir respuesta (U). La participante pasó el 18\% del día en un estado de ánimo e implicación positivo; el 5\% en un nivel +3 y otro 5\% en un nivel +5. No obstante, pasó gran parte del día (59\%) en un estado de ánimo e implicación negativo y un 14\% en un nivel -3. Esta participante registró 4 periodos ininterrumpidos de valores ME negativos (UNME) en la categoría Y, siendo el quinto periodo interrumpido para comer, aunque después volvió a encontrarse en malestar.

Observaciones generales para el personal: La participante estuvo en un potencial de implicación positiva del $100 \%$ en las categorías $F$ (ingesta), $P$ (asistencia práctica), T (estimulación directa de los sentidos) e Y (hablar solo). No obstante, su diversidad ocupacional fue tan sólo de 1 , en la categoría Y. En el estado en el que la participante se ha encontrado en +5 , tras recibir una asistencia, no se recogió suficiente información para conocer los motivos del mismo. Por otro lado, estuvo en un estado de agitación y angustia del 64\% en categoría Y. Sin embargo, los valores ME positivos transcurrieron cuando las auxiliares emprendían contacto (por ejemplo, dar un beso) con la participante. Es decir, el contacto con las auxiliares ha disminuido de forma positiva los estados de ansiedad y agitación pasados en la misma. Por esta razón, sería muy favorable seguir fomentando este tipo de relaciones personales para así incrementar su bienestar y, por lo tanto, su calidad de vida. 
BÚSQUEDA - Julio / Diciembre de 2014 - No. 13 (82 - 99)

\section{k) Resumen individual participante 11:}

Comentarios sobre los estados de ánimo e implicación, los comportamientos y el bienestar: La participante pasó parte del día (33\%) implicada pasivamente (B) y otro 33\% durmiendo (N). Sería aconsejable reducir la cantidad de ambos códigos. El 14\% lo pasó interactuando con los demás $(A)$; en concreto, pedía un vaso de agua; otro 14\% con autoestimulación repetitiva (W) y el $5 \%$ comiendo (F). Asimismo, estuvo el $79 \%$ del día en un estado de ánimo e implicación positivo. No obstante, pasó el 21\% del día en un estado de ánimo e implicación negativo en un nivel -5.

Observaciones generales para el personal: La participante estuvo en un potencial de implicación positiva del $19 \%$, pasado en las categorías A (interacción con los demás) y $F$ (ingesta). Su diversidad ocupacional tan sólo fue de 1, en la categoría A. Por otro lado, tuvo un aislamiento del $33 \%$ pasado en la categoría $N$ (dormir). ¿Podemos saber por qué se duerme cada veinte minutos? ¿Puede ser debido a un exceso de medicación? O quizás ¿por insomnio? O ¿aburrimiento? ¿Se podría ofrecer una implicación más activa? Los mayores niveles de valores ME positivos ocurrieron sobre todo cuando estaba implicada pasivamente y cuando se autoestimulaba de forma repetida. Los mayores niveles de valores ME negativos transcurrieron cuando en varias ocasiones la participante pedía un vaso de agua y se lo daban con espesante (se enfadaba mucho). ¿Realmente es necesario que beba agua con espesante? Si lo es ¿se pueden utilizar estrategias para distraerla y que acepte hidratarse?

\section{I) Resumen individual participante 12:}

Comentarios sobre los estados de ánimo e implicación, los comportamientos y el bienestar: La participante pasó gran parte del día (62\%) implicada pasivamente (B). ¿Se podría trabajar más en implicarla activamente? Para la próxima observación sería deseable haber reducido la cantidad de códigos pasados en esta categoría. Además, pasó el 14\% del día interactuando con los demás $(\mathrm{A})$; quejándose a la $\mathrm{AE}$ de un dolor en el lado izquierdo; el 10\% durmiendo $(\mathrm{N})$; otro $10 \%$ intentándose comunicar sin recibir respuesta $(U)$, expresando que "le duele el culo", y el $5 \%$ con periodos relacionados con la excreción $(X)$, para que la llevan hasta el baño. También la participante estuvo el $74 \%$ del día en un estado de ánimo e implicación positivo; no obstante, tuvo un estado de ánimo e implicación negativo del $5 \%$, además del $16 \%$ en un nivel -3 y el $5 \%$ en -5 .

Observaciones generales para el personal: La participante tuvo un potencial de implicación positiva del $14 \%$, pasado en la categoría A. Su diversidad ocupacional fue solamente de 1 , pasado en esta misma categoría. Por otro lado, estuvo en un estado de agitación y angustia del $10 \%$ en la categoría U, intentándose comunicar sin recibir respuesta. Asimismo, tuvo un aislamiento del $10 \%$ pasado en $\mathrm{N}$ (dormir). Los mayores niveles de valores ME positivos ocurrieron cuando se encontraba implicada pasivamente (B) y cuando interactuaba con la auxiliar (A). Sin embargo, los mayores niveles de valores ME negativos transcurrieron cuando se quejaba de dolor y nadie la atendía $(U)$ o tardaban en hacerlo. Este dolor podría ser una forma de comunicar que quería ir al baño ya que, tras unos IT alternados en las que la participante se quejaba de "dolor en el culo" y del lado izquierdo, sin ser atendida en algunas ocasiones, la $A E$ finalmente observó si había orinado, si le molestaba el pañal y la llevó hasta el baño. No obstante, debemos observar que la participante tuvo un trato de infantilismo, dañando su autoestima cuando la AE le miró el pañal en la sala con los demás residentes. Además, esta observación tan detractora no fue recogida por los mapeadores, lo que puede indicar una falta de empatía hacia las personas con demencia. Por otro lado, ¿Estas situaciones de dolor podrían ser una forma de llamar la atención? O quizás ¿Sería necesario tomar medicación?.

\section{m) Resumen individual participante 13:}

Comentarios sobre los estados de ánimo e implicación, los comportamientos y el bienestar: La participante pasó gran parte del día (71\%) hablando sola (Y), el 14\% con una actividad creativa $(E)$, el $5 \%$ implicada pasivamente $(B)$, otro $5 \%$ comiendo $(F)$ y otro $5 \%$ con estimulación directa de los sentidos $(T)$. Además, estuvo el $62 \%$ del día en un estado de ánimo e implicación positiva y un $33 \%$ en un nivel +3 . Por otro lado, 
pasó un 5\% en un estado de ánimo e implicación negativo en un nivel -3 .

Observaciones generales para el personal: La participante tuvo un potencial de implicación positiva del $95 \%$, pasado en las categorías $E$ (actividad expresiva), $\mathrm{F}$ (ingesta), T (estimulación directa de los sentidos) e Y (hablar sola). Su diversidad ocupacional fue de 3 , en las categorías E, T e Y. Por otro lado, estuvo en un periodo de agitación y angustia del $5 \%$ pasado en la categoría Y. Los mayores niveles de valores ME positivos transcurrieron cuando hablaba sola, comía y estaba implicada en una actividad expresiva. ¿Podemos saber con quién estaba hablando supuestamente y de qué? Así, quizás pudiéramos establecer las herramientas necesarias para proporcionarle el mismo estado de bienestar.

Tras haber expuesto las observaciones pertinentes de cada participante, por último, presentamos la tabla que contiene los PD y los PE recogidos durante todos los mapas (observaciones). Este resumen nos permite tener una visión global de las necesidades psicológicas más trabajadas por el personal:

Tabla 3. Resumen general de PD y PE observados

\begin{tabular}{|l|c|c|c|c|}
\hline \multicolumn{1}{|c|}{$\begin{array}{c}\text { Necesidad } \\
\text { psicológica }\end{array}$} & $\begin{array}{c}\text { Muy } \\
\text { detractor }\end{array}$ & Detractor & Potenciador & $\begin{array}{c}\text { Muy } \\
\text { potenciador }\end{array}$ \\
\hline Confort & & & 10 & \\
\hline Identidad & & & & \\
\hline Apego & & 2 & & \\
\hline Ocupación & & 2 & 3 & \\
\hline Inclusión & & & & \\
\hline
\end{tabular}

Fuente: elaboración propia

\section{Discusión}

En primer lugar, es necesario centrar nuestra atención en los resultados obtenidos en la tabla de detractores y potenciadores personales. Como podemos observar, el confort es la necesidad más registrada en el centro. El suministro de cariño y proximidad a los demás han sido los aspectos más trabajados, sin embargo, no han resultado suficientes, puesto que se han proporcionado en muy pocos casos. Otro de los aspectos que recoge el confort es el consuelo y la ternura que en ninguno de los casos se ha ofrecido. Por ello, nos preguntamos ¿Qué provoca esta situación? ¿La falta de personal? ¿De tiempo? ¿Burnout? ¿Falta de implicación con los usuarios?

Por otro lado, se han trabajado las necesidades psicológicas de apego y ocupación. Sin embargo, del mismo modo que la variable de confort, la presencia de ambas sigue siendo muy escasa e incluso, detractoras. Además, debemos trabajar para que la implicación pasiva no se convierta en una falta de implicación ¿Cómo podemos intervenir para mejorar su bienestar?.

En cuanto a las necesidades de identidad e inclusión, éstas no se han observado, lo que significa que hay que trabajar en proporcionar un entorno idóneo para que los participantes se encuentren a sí mismos. Una manera de hacerlo podría ser incluyendo más actividades a los residentes de lasala, como el uso de flores y juegos de pelota. Incluso podría ponerse música, así se podría estudiar el valor del contacto sensorial con los usuarios. Asimismo, se podrían introducir actividades como las terapias alternativas como por ejemplo, risoterapia, aromaterapia... y de este modo, evitar su desvinculación. 
BÚSQUEDA - Julio / Diciembre de 2014 - No. 13 (82 - 99)

También, para mejorar las relaciones personales se podrían buscar maneras de relacionarse entre ellos mismos con el apoyo del personal, creando grupos de autoayuda, grupos de trabajo, grupos de encuentro o dinámicas grupales. Acciones que irían encaminadas a desarrollar el empoderamiento y la mayor implicación de las personas mayores con menor afectación y que ayudarían a retrasar el deterioro que a menudo acelera el aislamiento, el aburrimiento, la tristeza y la soledad, ocasionados por la institucionalización.

Otra forma que existe de relacionarse sería propiciar encuentros con estudiantes, tanto de instituto como de universidad, niños, voluntarios... Un ejemplo de intervenciones en este sentido son las que se están llevando a cabo en Estados Unidos desde hace unos cuantos años: se trata de los programas intergeneracionales. Según HattonYeo y Ohsako (2001; citado en Sánchez, 2007): "Los programas intergeneracionales son medios para el intercambio intencionado y continuado de recursos y aprendizaje entre las generaciones mayores y las más jóvenes con el fin de conseguir beneficios individuales y sociales" (p.38).

Algunos de estos programas intergeneracionales se llevan a cabo en los centros de día para personas con Alzheimer como es el caso de Neighbors Growing Together en Virginia, un programa dirigido desde la Facultad de Psicología de la Virginia Tech University que une a personas mayores con niños de 0-3 años. Entre los beneficios evaluados de estos programas se han encontrado:

- Beneficios intrapsíquicos e individuales: Mayor implicación en las actividades; mayor emisión de la comunicación verbal (CV) y comunicación no verbal (CNV); aumento de la motivación por las actividades; responsabilidad por el cuidado (sentimiento de utilidad); actividades con significado (autoestima); disminución del estrés; disminución de las conductas problemáticas: ira, agresividad.

- Beneficios educativos y terapéuticos: disfrute con actividades de psicomotricidad fina y gruesa; preservación de capacidades cognitivas; realización de actividades presentes en la memoria a largo plazo.

También pueden participar personas con enfermedades a largo plazo como el cáncer, personas con VIH/SIDA o la enfermedad del Parkinson; personas que han tenido un ictus; cuidadores de personas mayores o muy jóvenes, bebés y niños de seis semanas hasta seis años; jóvenes en riesgo (incluyendo niños de crianza y padres adolescentes); jóvenes y adultos con discapacidad y, en definitiva, personas de todas las edades que quieran formar parte de este enfoque intergeneracional.

De esta manera, cuando las personas mayores y niños participan conjuntamente en las actividades, las personas mayores recuerdan canciones, juegos y proyectos de su pasado, puesto que comparten sus conocimientos, paciencia y experiencia con niños en edad preescolar.

En otros casos, los jóvenes acuden a los centros de día o residencias y hacen que las personas mayores se sientan más cómodas con las nuevas tecnologías, mientras que éstas favorecen en un mayor desarrollo las habilidades de los jóvenes (como enseñanza de actividades manuales propias de una región: cuero, cestería..., manualidades que muchas personas mayores dominan). Su objetivo es relacionarse recíprocamente, basándose en el respeto mutuo, en el dar y el recibir en un contacto significativo y, por lo tanto, desarrollar un sentido de comunidad.

En segundo lugar, es importante resaltar que una de las observaciones que más se ha contemplado es la falta de implicación de los usuarios con el entorno, por lo que nos hemos preguntado si esto se debía a una falta de personal, de tiempo, burnout... Además de estos factores, la falta de formación en la atención adecuada a los procesos de demencia (tipos de demencia, atención a los comportamientos difíciles, motivación para la implicación en actividades de las personas con Alzheimer u otras demencias, programas 
intergeneracionales, terapias no farmacológicas, tratamiento individualizado, atención centrada en la persona...) es un valor añadido. Es necesario que los profesionales que intervienen estrechamente con la persona (psicólogos, trabajadores sociales, auxiliares, personal de enfermería y de medicina, educadores sociales, TASOC...) tengan las herramientas adecuadas para tratar la demencia y poder retrasarla así, desarrollando las intervenciones pertinentes.

Por ello, es muy importante empezar concienciando a los poderes políticos (a través de investigaciones, publicaciones, congresos y jornadas) de lo que significa y requiere una atención centrada en la persona (ACP) para que éstos empiecen a tener en cuenta a las personas con demencia. Como bien explican Brooker y Surr (2009): Para influir en las prioridades políticas, hemos de ser capaces de explicar qué significa ACP, tanto a nivel práctico como en la investigación. Este tipo de atención no es solo sinónimo de asesoramiento o atención individualizados, sino que se ha convertido en un término que engloba todo un movimiento en el campo de la demencia que trasciende esos dos conceptos.

En tercer lugar y a modo general, es necesario hacer énfasis en unos factores de riesgos referentes a las úlceras por presión (UPP en adelante) que no deben olvidarse. Hemos podido observar que no se lleva a los participantes suficientemente al baño y, puesto que la mayoría tiene incontinencia y síndrome de inmovilidad, es evidente que deberían ser supervisados más a menudo. Si sus heces permanecen mucho tiempo en el pañal hasta que una auxiliar lo ve y lo cambia podemos encontrarnos ante un riesgo de UPP, debido a que la piel se mantiene húmeda y sin la higiene correcta. Por ello, deben trabajarse más maneras de prevenirlas, ya sea promoviendo su movilidad; comprobando más a menudo si hay que cambiarle el pañal, manteniendo una higiene correcta, etc. Por otro lado, debemos tener en cuenta que una mal nutrición también puede ser sinónimo de úlceras, lo que resulta impactante que en un centro residencial haya personas desnutridas.
En cuarto lugar y último, es necesario destacar ciertas limitaciones del instrumento y del estudio en sí. Una de ellas es que no se han recogido suficientes valores ME a lo largo de cuatro horas o 48 IT, tal y como recomienda el manual del DCM, lo cual no ha permitido elaborar un perfil de bienestar/malestar (WIB) de los participantes. Ello podría ser consecuencia del exceso de trabajo o simplemente, la poca variabilidad existente en la dinámica de la residencia, lo cual confirma la poca actividad y estímulos existentes en el centro en cuanto a la atención a personas con demencia. Sin embargo, este hecho no ha condicionado que se tengan en cuenta otros factores que puedan propiciar mayor calidad de vida.

Como se ha mencionado en varias ocasiones, el DCM recopila datos mediante la observación de personas con demencia que viven en centros de atención especializada. En este sentido, es necesario hacer énfasis en que las observaciones solo tienen lugar en las áreas comunes de los centros asistenciales, con lo cual el DCM no aporta información sobre la atención que los participantes reciben en los dormitorios y cuartos de baño, por lo que subestima los cuidados íntimos y físicos que se ofrecen en los centros. Principalmente, el DCM nos aporta datos del entorno psicosocial de los participantes, no obstante, la información sobre la influencia de los cuidados físicos se recaba de manera indirecta.

Otro aspecto importante a destacar de esta herramienta es que, en otras actualizaciones del DCM, no era adecuada su utilización para las personas con una demencia avanzada. Sin embargo, en la última actualización (DCM 8), se intentaron ajustar los códigos para que también sean válidos para las personas con una demencia más avanzada. De esta manera, el uso del DCM puede ayudar a comprender algunas cuestiones a nivel individual y a valorar los progresos. 
BÚSQUEDA - Julio / Diciembre de 2014 - No. 13 (82 - 99)

\section{Conclusiones}

A raíz de los resultados anteriormente analizados, podemos señalar que, principalmente, debemos estimular las capacidades en constante desuso de las personas mayores con demencia, orientándolas en el tiempo, espacio y persona. Asimismo, mantener su independencia en las actividades de la vida diaria, atendiendo a las necesidades más personales e individuales. La conservación de la persona en el contexto de las relaciones personales, muy favorables para mantener un bienestar relativo en la demencia, es de vital importancia.

Diferentes estudios han demostrado que no existe correlación entre el grado de deterioro cognitivo y los niveles de bienestar/malestar de las personas con demencia, es más, se sugirió que bajas puntuaciones en estos niveles junto con altos niveles de dependencia podían relacionarse más bien con una peor calidad de atención psicosocial (Edelman et al., 2004; Jarrot \& Bruno, 2003; Gigliotti et al., 2004, citados en Brooker \& Surr, 2009).

Por estos motivos, el uso del DCM es una herramienta que puede optimizar la atención ofrecida en una institución y orientarla hacia un enfoque más individualizado y no tan centrado en las labores que se desarrollan en ella. Por ello, es importante introducir el concepto de la atención centrada en la persona en todas las organizaciones de carácter asistencial, incidiendo en la necesidad de aplicar métodos que mejoren la calidad de vida de las personas que están atendiendo, ya que son su principal objetivo. Tal y como señala Brooker y Surr (2009), las horas de comida, las actividades o los cambios de turno, a menudo revelan qué cosas pueden cambiarse para mejorar el bienestar de todos los usuarios del servicio.

El DCM es una escala que mide la calidad de vida tanto de la persona con demencia como de la calidad asistencial recibida en un intervalo de tiempo mayor al que otras escalas tienen, como por ejemplo, la escala FUMAT (Verdugo,
Gómez \& Arias, 2008), la cual tiene una duración de 10 minutos, un periodo de tiempo apto para la poca disponibilidad de los profesionales, pero insuficiente para mostrar verídicamente la calidad de vida de una persona. De la misma forma ¿podríamos conocer la calidad de vida que pueden ofrecer unos padres a un hijo en proceso de adopción en 10 minutos? En este estudio las observaciones realizadas se balancean en un intervalo de 2 a 4 horas, siendo el motivo de este corto periodo de tiempo la inactividad de la persona objeto de estudio.

Finalmente, hacer énfasis en la necesidad de continuar trabajando para seguir manteniendo las capacidades de las personas mayores, ya que si estas no se mantienen trabajando al final se acaban perdiendo. EI DCM aporta muchos datos sobre cómo optimizar el bienestar de una persona a lo largo del día, puesto que las pequeñas cosas que provocan alegría o inquietud, normalmente se pasan por alto en los planes de atención general (Brooker \& Surr, 2009).

Las personas luchamos día a día por nuestra autodeterminación, por seguir manteniendo una participación activa en aquellas áreas donde nos sea posible, por seguir tomando nuestras propias decisiones con independencia del grado de capacidad funcional que tengamos. Señalar qué tipo de servicios requerimos, qué tipo de participación deseamos mantener y con quién..., son algunas de las decisiones que queremos seguir tomando a lo largo de nuestra vida. Las personas mayores son capaces de contribuir de manera activa y ser apoyo a sus familias y a la comunidad en la que viven (Pinazo, 2011) y éste es uno de los objetivos que no se deben perder de vista.

En todos los países donde se utiliza el DCM cada vez son más los que apuestan por una atención que cubra no solo las necesidades físicas, sino también las emocionales y que esté centrada más en la persona que en las actividades a realizar (Brooker \& Surr, 2009). Actualmente, el DCM se utiliza en todo el mundo y está consolidado en muchos países. 
Marta Sahagún Navarro - Atención de personas mayores con demencia a persona en centros residenciales

Hoy por hoy, nos encontramos ante entornos que ofrecen pocas oportunidades para la implicación y relación con otras personas y/o actividades, lo que conlleva una radical despersonalización de las personascon demencia y el refuerzo de la percepción de la sociedad en cuanto a que éstas no son del todo humanas. Por ello, identificar estas características, resulta el elemento indispensable para transformarlas y conseguir que las relaciones y los entornos de cuidados sean personalizadores y refuercen todo aquello que otorga estatus a una persona (Barnett, 2000; Brooker \& Surr, 2005; Sabat, 1994, citado en Vila-Miravent et al., 2012).

\section{Referencias bibliográficas}

Alberdi, I. (1999). La nueva familia española. Madrid: Taurus.

Bradford Dementia Group (2009). DCM 8. Manual del usuario. Barcelona: Fundació Alzheimer Catalunya.

Brooker, D. (2006). Dementia-Care Mapping. Visión general y resumen de la bibliografía de investigación. Alzheimer. Realidades e investigación en Demencia, 33.

Brooker, D. \& Surr, C. (2009). Dementia-Care Mapping. Principios y práctica. Barcelona: Fundació Alzheimer Catalunya.

Fernández, E. \& Vila, J. (2006). ¿Qué es una asistencia de calidad para las personas con demencia? Alzheimer. Realidades e investigación en Demencia, 33.

Fernández, E. \& Vila, J. (2006). Dementia-Care Mapping (DCM). Dosier informativo. Recuperado el 10 de Agosto de 2011 de http://www.inforesidencias.com/docs/ dementia_care_maping.pdf

Instituto de Mayores y Servicios Sociales (2009). Las personas mayores en España. Datos estadísticos estatales y por Comunidades Autónomas. Informe 2008. Recuperado el 23 de Agosto de 2011 de http://www. imserso.es/InterPresent1/groups/imserso/ documents/binario/infppmm2008.pdf

Julve, M. (2011). Modelos de protección social a la dependencia. Apuntes del Máster en Atención Sociosanitaria a la Dependencia. Material fotocopiado.

Julve, M. (2011). Valoración de la situación de dependencia. Apuntes del Máster en Atención Sociosanitaria a la Dependencia. Material fotocopiado.

Montorio, I. (2007). Intervenciones psicológicas en la prevención y atención a la dependencia. Intervención Psicosocial, 16(1), 43-54.

Pinazo, S. (Dir.) (2011). Autonomía personal y dependencia. Bases teóricas y práctica social. Valencia: Publicaciones de la Universitat de València.

Sánchez, M. (Dir.), Butts, D.M., Hatton-Yeo, A., Henkin, N.A., Jarrott, S.E., Kaplan, M.S., Martínez, A., Newman, S., Pinazo, S., Sáez, J. \& Weintraub, A.P.C. (2007). Programas intergeneracionales. Hacia una sociedad para todas las edades. Colección Estudios Sociales, 23.

Verdugo, M. A., Gómez, L. E. \& Arias, B. (2009). Evaluación de la calidad de vida en personas mayores: La Escala FUMAT. Salamanca: Instituto Universitario de Integración en la Comunidad.

Vila-Miravent, J., Villar, F., Celdrán, M. \& Fernández, E. (2012). El modelo de la atención centrada en la persona: análisis descriptivo de una muestra de personas mayores con demencia en centros residenciales. Revista de Psicologia, Ciències de l'Educació i de l'Esport, 30(1), 109-117. 